\title{
Imaging microvascular dynamics noninvasively with realtime photoacoustic microscopy
}

Roger Zemp, Rachel Bitton, Meng-Lin Li, K. Kirk Shung, Lihong V. Wang

Roger Zemp, Rachel Bitton, Meng-Lin Li, K. Kirk Shung, Lihong V. Wang, "Imaging microvascular dynamics noninvasively with realtime photoacoustic microscopy," Proc. SPIE 6430, Advanced Biomedical and Clinical Diagnostic Systems V, 643015 (13 February 2007); doi: 10.1117/12.700484

SPIE. Event: SPIE BiOS, 2007, San Jose, California, United States 


\title{
Imaging Microvascular Dynamics Noninvasively with Realtime Photoacoustic Microscopy
}

\author{
Roger Zemp ${ }^{a}$, Rachel Bitton ${ }^{b}$, Meng-Lin Li $^{c}$, K. Kirk Shung ${ }^{b}$, and Lihong V. Wang ${ }^{a}$ \\ ${ }^{a}$ Optical Imaging Laboratory, Department of Biomedical Engineering, Washington University, \\ St. Louis, MO, 63130 \\ ${ }^{b}$ Department of Biomedical Engineering, University of Southern California, Los Angeles, CA, \\ 90089 \\ ${ }^{c}$ Dept. of Electrical Engineering, National Tsing Hua University, Hsinchu, 30013, Taiwan.
}

\begin{abstract}
A realtime photoacoustic microscopy system consisting of a high-repetition rate pulsed laser, high-frequency (30 $\mathrm{MHz})$ ultrasound array transducer, and realtime receiving system was used to visualize microvessels pulsations over a cardiac cycle. The system offers $100 \mu \mathrm{m}$ lateral spatial resolution, $25 \mu \mathrm{m}$ axial spatial resolution, and can image at a rate of 83 frames per second. The system shows promise for visualizing time-varying processes in the microvasculature.
\end{abstract}

\section{INTRODUCTION}

High-resolution optical microscopy has revealed much about our natural world, however microscopy and coherencebased imaging techniques are fundamentally depth-limited due to the multiple scattering of light in optically turbid tissue. Photoacoustic microscopy is a novel imaging technology for imaging absorbing structures in turbid media and can overcome the transport mean-free path depth limitation of traditional microscopy by relying on ultrasonic detection of photoacoustic signals for spatial resolution.

Photoacoustic microscopy is emerging as a powerful tool for non-invasively visualizing microvascular networks in vivo. ${ }^{1,2}$ Photoacoustic signals are generated when laser pulses are absorbed in optically-absorbing structures causing a rapid thermoelastic expansion. Fortuitously, oxy-hemoglobin and deoxy-hemoglobin are the dominant absorbing substances in the body, hence high contrast images of blood vessels are possible. Besides offering structural information about vessel morphology, illumination with multiple optical wavelengths can be used to estimate local blood oxygenation.

Thus far, research in photoacoustic microscopy has largely focused on imaging static microvascular structure or slow time-varying trends in blood oxygenation. This letter reports a first-of-its kind realtime microvascular imaging system, and its utility in visualizing microvascular dynamics.

The microvasculature is known to be a highly dynamic system. Smooth muscle in arterioles play an important role in vasodilation and vasoconstriction in response to chemical stimuli, and regulating the supply of blood and nutrients to tissues in response to metabolic demand. Following tissue injury or inflammation, microvessels are thought to vasodilate and become more porous to allow neutrophils and macrophages to migrate to the site of injury, and to allow enhanced nutrient supply in the wound-healing process. Impairment of such microvascular function may be a key to some diseases.

Microvascular dynamics over a cardiac cycle are also of great interest. Following systole, a surge of blood from the aorta propagates through the arteries and is driven through arterial bifurcations into microvascular networks where, due to greater impedance, the pulse wave is reflected back along the arteries to the heart. The reflected pulse wave was discovered to play an important role in supplying blood to the coronary arteries. In atherosclerosis, the viscoelastic properties of arteries changes which can significantly alter these important cardiovascular pulse-wave dynamics. The dynamics of these pulse waves in the microvasculature in health and disease is poorly understood, although it is thought that atherosclerosis and other diseases can adversely affect the viscoelastic properties and pulse-wave impedance of the microvasculature.

Unfortunately, there are few tools for studying microvascular dynamics non-invasively. Invasive techniques for studying microvascular function include cannulation, and epifluorescence (or other) microscopy approaches for

Advanced Biomedical and Clinical Diagnostic Systems $V$

edited by Tuan Vo-Dinh, Warren S. Grundfest, David A. Benaron, Gerald E. Cohn, Ramesh Raghavachari

Proc. of SPIE Vol. 6430, 643015, (2007) · 1605-7422/07/\$18 - doi: 10.1117/12.700484

Proc. of SPIE Vol. $6430643015-1$ 
visualizing surgically-exposed microvessels in animal models. While high-field contrast-enhanced MRI is capable of visualizing small vessels in vivo, to our knowledge, visualization of cardiovascular-induced microvascular dynamics have not yet been demonstrated. High-frequency Doppler ultrasound ${ }^{3,4}$ has shown promise in visualizing flow in arterioles and venules, however, its success is conditioned on non-trivial clutter filters and vessels with slow flow velocities may be missed. Optical coherence tomography has shown promise in visualizing microvascular oscillations as well as functional response of microvessels, however, it is fundamentally depth-limited to approximately $1 \mathrm{~mm}$, precluding many interesting studies in deeper subdermal layers. Otoscopes are frequently used by physicians in diagnosing hypertention, retinopathy, glaucoma, and other diseases by visualizing the arterioles and venules on the retinal surface. Microvascular pulsations are visible with this simple technology. Early detection of abnormal microvascular dynamics and morphology in other non-transparent anatomical locii could prove important in diagnostic and interventional strategies. Additionally, microvascular imaging technologies may provide a much needed tool for basic research and for monitoring therapeutic efficacy in vivo.

Here we report on a novel photoacoustic microscopy system which has the potential to address some of these needs. Our system uses a $30 \mathrm{MHz}$ ultrasound array transducer. Advantages of this array over a mechanically scanned single element transducer include ability to use dynamic receive focusing (greatly increasing the depthof-field), electronic rather than mechanical B-scanning capability, and parallel data acquisition. Eventually the probe may be handheld and may provide realtime imaging capabilities. Other groups are beginning to use arrays for photoacoustic imaging ${ }^{5}$ but this is the first system using high frequency (above $20 \mathrm{MHz}$ ) photoacoustic imaging of the microvasculature. We report further progress from results recently reported. ${ }^{6,7}$

Due to small detection area of individual elements and difficulties in constructing high-frequency electronics, there may be some question regarding whether high frequency arrays are sufficiently sensitive for imaging in vivo. We show feasibility of the high-frequency array system for photoacoustic visualization of microvessels. It is anticipated that systems similar to the one reported here may be used for studying and diagnosing a number of diseases where microvascular changes impact health, including cancer ${ }^{8}$ dermatological disorders, cardiovascular disease, and peripheral microvascular complications in diabetics. We demonstrate visualization of microvascular pulsations by photoacoustic microscopy. The basic premise of visualizing the pulse-waves in the microvasculature is that the photoacoustic signal amplitude may change with vessel diameter changes due to increased local blood volume. High-frequency photoacoustic imaging may complement high-frequency Doppler ultrasound ${ }^{3,4}$ to detect very small vessels as well as those with low flow velocities.

\section{BEAMFORMING}

Channel data was acquired at $250 \mathrm{MS} / \mathrm{s}$ and processed using a sector-scan beamforming algorithm modified from ultrasound beamforming. To reconstruct an A-scan line along a line of sight defined by the angle $\theta$, we apply dynamic delays $\tau_{n}(R, \theta)$ to the received channel data $S(n, t), n=1,2, \ldots, 48$, where $R$ is the distance from the image point to the beamforming origin (taken here as the center of the array) then add the delayed data. The time delays are computed from geometric considerations. The time-of flight along a path distance between a field point and array element $x_{n}$ is given as

$$
\begin{aligned}
D(n, R, \theta) & =\sqrt{\left(x_{n}-R \sin \theta\right)^{2}+R^{2} \cos ^{2} \theta} \\
& =R \sqrt{1+\frac{x_{n}^{2}}{R^{2}}-2 \frac{x_{n}}{R} \sin \theta} .
\end{aligned}
$$

where $x_{n}$ is the distance to the array element from the array center, and $c$ is the speed of sound. Required element delays for dynamic focusing are derived by taking a second-order Taylor approximation in $x_{n}$ and subtracting the propagation delay $R / c$ to obtain

$$
\tau_{n}(R, \theta)=-\frac{x_{n} \sin \theta}{c}+\frac{x_{n}^{2} \cos ^{2} \theta}{2 R c},
$$

which is effective whenever the Fresnel approximation holds. 


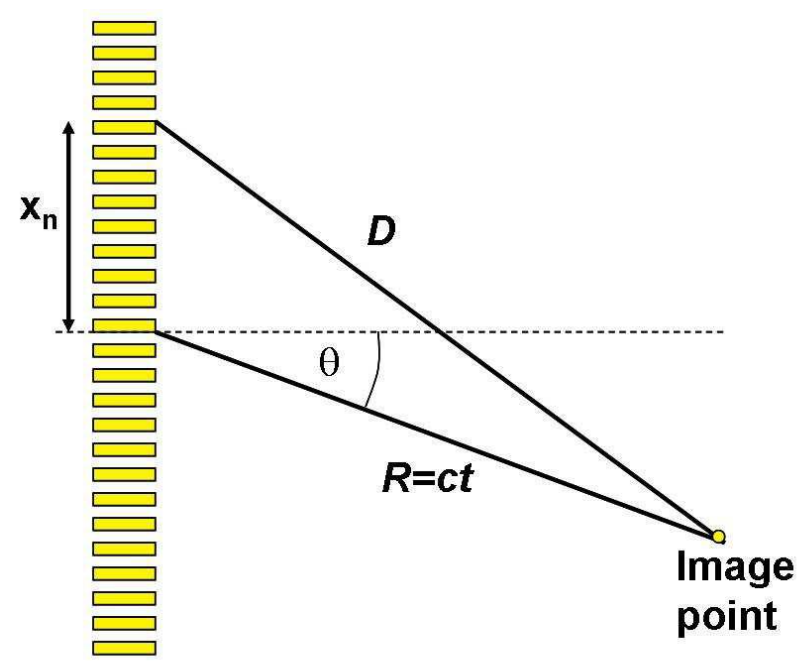

Figure 1. Beamforming geometry.

The first term of (2) represents steering, while the second represents focusing. The transit time between a point of interest and the array for pulse-echo ultrasound is $t=2 R / c$, while in photoacoustic imaging propagation is one-way, and $t=R / c$. The time delay applied to element $n$, for sector-scan angle $\theta$ is thus given as

$$
\tau_{n}(t, \theta)=-\frac{x_{n} \sin \theta}{c}+\frac{x_{n}^{2} \cos ^{2} \theta}{2 t c^{2}},
$$

Hence the steering term $-\frac{x_{n} \sin \theta}{c}$ is identical to pulse-echo ultrasound, while the focusing term $\frac{x_{n}^{2} \cos ^{2} \theta}{2 c^{2} t}$ is different. In beamforming, we used all 48-elements to leverage the maximum possible aperture. We also found it advantageous to apply a coherence factor $(\mathrm{CF})$ weighting in the beamforming procedure. ${ }^{9}$ A coherence factor for an A-scan line with direction $\theta_{m}$ is given as

$$
C F\left(t, \theta_{m}\right)=\frac{\left|\sum_{n=0}^{N-1} S\left(n, t-\tau_{n}\left(R, \theta_{m}\right)\right)\right|^{2}}{N \sum_{n=0}^{N-1}\left|S\left(n, t-\tau_{n}\left(R, \theta_{m}\right)\right)\right|^{2}},
$$

where $N$ is the number of elements. This time-varying function is applied multiplicatively to each beamformed A-scan and tends to emphasize highly in-phase signals while rejecting low coherence side-lobes, grating lobes, and noise.

\section{PHOTOACOUSTIC SIGNAL DEPENDENCE ON MICROVASCULAR DIAMETER}

Dynamic changes in microvessel diameter are expected to produce changes in the photoacoustic signal amplitude due to greater local blood volume. This enhancement can be described analytically, as described next.

\subsection{Photoacoustic Signals from a Uniformly-Illuminated Optically-Absorbing Infinite Cylinder: Analytic Solution}

We can modeling a blood vessel as an effectively infinite optically absorbing cylinder which has been uniformly illuminated. Neglecting acoustic attenuation and dispersion, the photoacoustic pressure $p_{f}$ at a normal distance $r$ from an infinite cylinder of radius $a$ with optical absorption coefficient $\mu_{a}$, due to a delta-function optical pulse with energy $E_{o}$ has been shown to have the closed form: ${ }^{10}$

$$
p_{f}(\hat{\tau})=\frac{\kappa}{2 \pi} \sqrt{\frac{\pi a}{r}}
$$




$$
\begin{aligned}
& \times \quad\left[\frac{\Gamma\left(\frac{3}{4}\right)}{2^{1 / 2} \Gamma\left(\frac{5}{4}\right)} F_{1}\left(\frac{3}{4},-\frac{1}{4}, \frac{1}{2},(\hat{\tau}-1)^{2}\right)\right. \\
& \left.-\quad 2^{1 / 2} \frac{\Gamma\left(\frac{5}{4}\right)}{\Gamma\left(\frac{3}{4}\right)}(\hat{\tau}-1) F_{1}\left(\frac{5}{4}, \frac{1}{4}, \frac{3}{2},(\hat{\tau}-1)^{2}\right)\right]
\end{aligned}
$$

for $0<\hat{\tau}<2$, and

$$
\begin{aligned}
p_{f}(\hat{\tau}) & =-\frac{\kappa}{2 \pi} \sqrt{\frac{\pi a}{r}} \frac{\Gamma\left(\frac{3}{2}\right)}{2^{3 / 2} \Gamma(2)}(\hat{\tau}-1)^{-3 / 2} \\
& \times\left[F_{1}\left(\frac{3}{4}, \frac{5}{4}, 2,(\hat{\tau}-1)^{-2}\right)\right. \\
& \left.+\quad F_{1}\left(\frac{5}{4}, \frac{3}{4}, 2,(\hat{\tau}-1)^{-2}\right)\right]
\end{aligned}
$$

for $\hat{\tau}>2$, and zero for $\hat{\tau}<0$, with $\hat{\tau}=\frac{c}{a}\left(t-\frac{r-a}{c}\right)$ defined as the retarded time from the closest edge of the cylinder. Here $c$ is the speed of sound in the medium, assumed equal to the speed of sound in the cylinder, and $t$ is time. $\kappa$ is defined as

$$
\kappa=\mu_{a} E_{o} \beta c^{2} / C_{P}
$$

where $\beta$ is the thermoelastic expansion coefficient and $C_{P}$ is the specific heat. The above solution assumes that the acoustic impedance and speed of sound of the cylinder and the medium are identical.

The maximum amplitude occurs just after time $\hat{\tau}=0$, corresponding to the signal from the point of the cylinder closest to the observation point. It can be observed that the maximum pressure $p_{m} \equiv \max _{\hat{\tau}}\left\{p_{f}(\hat{\tau})\right\}$ in this case is proportional to the square root of the cylinder radius:

$$
p_{m} \propto \sqrt{\frac{a}{r}}
$$

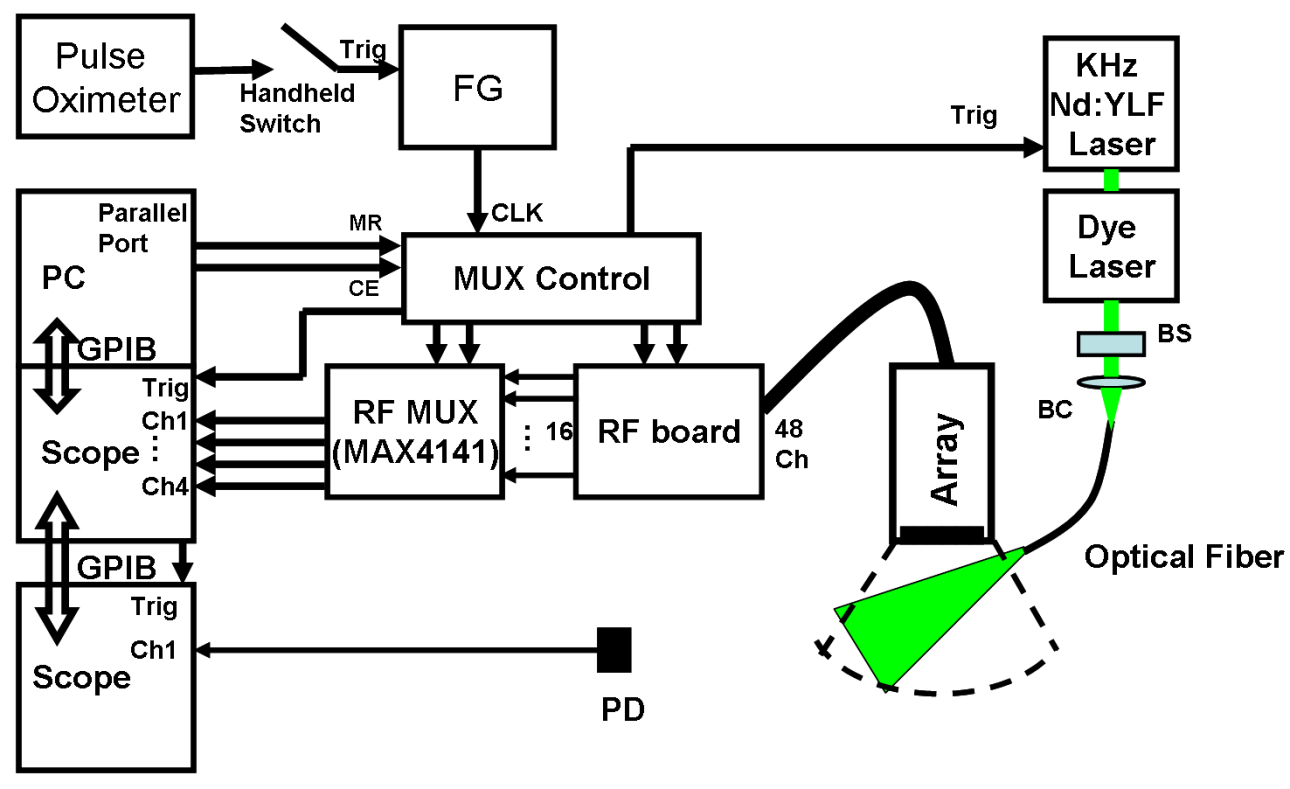

Figure 2. System diagram. GG: ground-glass diffuser, L: lens, MUX: multiplexer. 


\section{SYSTEM DESCRIPTION}

A novel photoacoustic microscopy system was developed for visualizing these microvascular dynamics. Our system, shown in Fig. 1 consists of a high-frequency ultrasound array, a high pulse-repetition rate laser system, and receiving electronics. The ultrasound transducer is a $30 \mathrm{MHz}$ piezo-composite array of 48 elements ${ }^{11}$ with $50 \%$ pulse-echo -6dB fractional bandwidth, $100 \mu \mathrm{m}$ pitch (2 times the center wavelength), $2 \mathrm{~mm}$ elevation aperture and $8.2 \mathrm{~mm}$ elevational focus, previously reported for photoacoustic microvascular imaging using a $10 \mathrm{~Hz}$ pulserate laser and slower data acquisition system. In this article we use a Nd:YLF Q-switched laser (INNOSLAB, Edgewave GmbH, Germany) to pump a high flow-rate dye laser (Cobra, Sirah Laser -und Plasmatechnik GmbH, Germany). The pumping laser used a continuous-wave beam rather than a flashlamp to excite the lasing cavity, which was gain-modulated by an electro-optic Q-switch. This novel design enabled the pump laser to produce $14 \mathrm{~mJ}$ pulses of $6.5 \mathrm{~ns}$ duration at $1 \mathrm{KHz}$ pulse-repetition frequencies (PRF). Higher PRFs were possible at the cost of pulse energy. Roughly $2 \mathrm{~mJ}$ of pulse energy was produced by the dye laser which was then coupled into a $600 \mu \mathrm{m} 0.39$ N.A. optical fiber using a beam shaper and microscope objective with free-space positioning system. The beam shaper was necessary to convert the elliptically-shaped beam to a circular shape before fiber coupling. A custom aperture truncated stray light before fiber-coupling. Approximately $0.7 \mathrm{~mJ}$ of fiber-coupled $566 \mathrm{~nm}$ light was delivered to the skin surface in an area of approximately 1.5 by $4 \mathrm{~mm}$, giving an estimated fluence of $12 \mathrm{~mJ} / \mathrm{cm}^{2}$, less than the $20 \mathrm{~mJ} / \mathrm{cm}^{2}$ permissible by ANSI standards. A fast photodiode was used to record the energy of each laser pulse to compensate for pulse-to-pulse amplitude fluctuations. The photodiode signal was recorded on an oscilloscope communicating with the host oscilloscope using GPIB communication protocols.

Signals from the array were amplified by a custom RF board with $33 \mathrm{~dB}$ fixed gain and an additional 40 $\mathrm{dB}$ of variable gain. The board multiplexed the 48-channels down to 16. Four 4-to-1 RF multiplexer boards (MAX4141) were then used to enable data acquisition with a 4-channel oscilloscope (Tektronix, TDS5034). Multiplexer control logic consisted of a custom asynchronous 4-bit binary counter looping from binary 0 to 11 (using two CD74HCT163E digital counter IC's), to sequentially select each of the 12 multiplexer states. Counting enable, counter preset enable, and master reset lines were controlled via the parallel-port on the PC portion of the oscilloscope. A D-flip-flop was also used pass the function generator trigger pulses on their rising edge to the oscilloscope and laser only when the count-enable line was high. MATLAB running on the oscilloscope controlled the data acquisition. The fast-frame mode of the oscilloscope allowed for roughly 200 records of 500 samples to be acquired at high $(\mathrm{KHz})$ trigger rates before data transfer and offline processing. The channel memory size allowed for 22 image frames (with 12 pulses per frame) to be acquired using 500 samples per record at $250 \mathrm{MS} / \mathrm{sec}$. Each image frame was acquired by pulsing the laser at $1 \mathrm{KHz}$ for 12 pulses, thus a frame could be acquired in $120 \mathrm{~ms}$, and the frame rate could be as high as 83 frames per second. This high frame-rate is appropriate for mice or rats who have heart rates of 200-400 beats per minute or 4-6 beats per second.

\section{RESULTS}

The system was shown to have lateral resolution of better than $100 \mu \mathrm{m}$ and axial resolution of $25 \mu \mathrm{m}$. We imaged a $6 \mu \mathrm{m}$ carbon fiber in distilled water with $1 \%$ intralipid at a depth of $7.2 \mathrm{~mm}$, using 40 times averaging, as shown in Fig. 5. The reduced scattering coefficient of this water-intralipid media was approximately $\mu_{s}^{\prime}=10 \mathrm{~cm}^{-1}$, which is close to biological tissue.

Vessels in a Sprague Dawley rat were visualized to depths of $3 \mathrm{~mm} .{ }^{7}$ In this article we used 8 frames per second to capture 5 image frames every cardiac cycle. A pulse oximeter (NONIN 8600V) provided a TTL trigger signal every cardiac cycle. After arming the oscilloscope, a handheld switch enabled the pulse oximeter to trigger

the function generator to fire a burst sequence for triggering image acquisition. The burst sequence consisted of 20 bursts of 12 TTL pulses (with $1 \mathrm{KHz}$ PRF) at $0.125 \mathrm{~s}$ intervals, leading to 20 frames of 5 images per cardiac cycle for 4 cardiac cycles. A movie of the microvessels shows pulsatile microvessel bulk displacements. Additionally, vessels showed visible amplitude changes over time. Two example image frames from diastole and systole are shown in Fig. 4, with the boxed region showing a vessel where there is a visible amplitude difference between image frames 


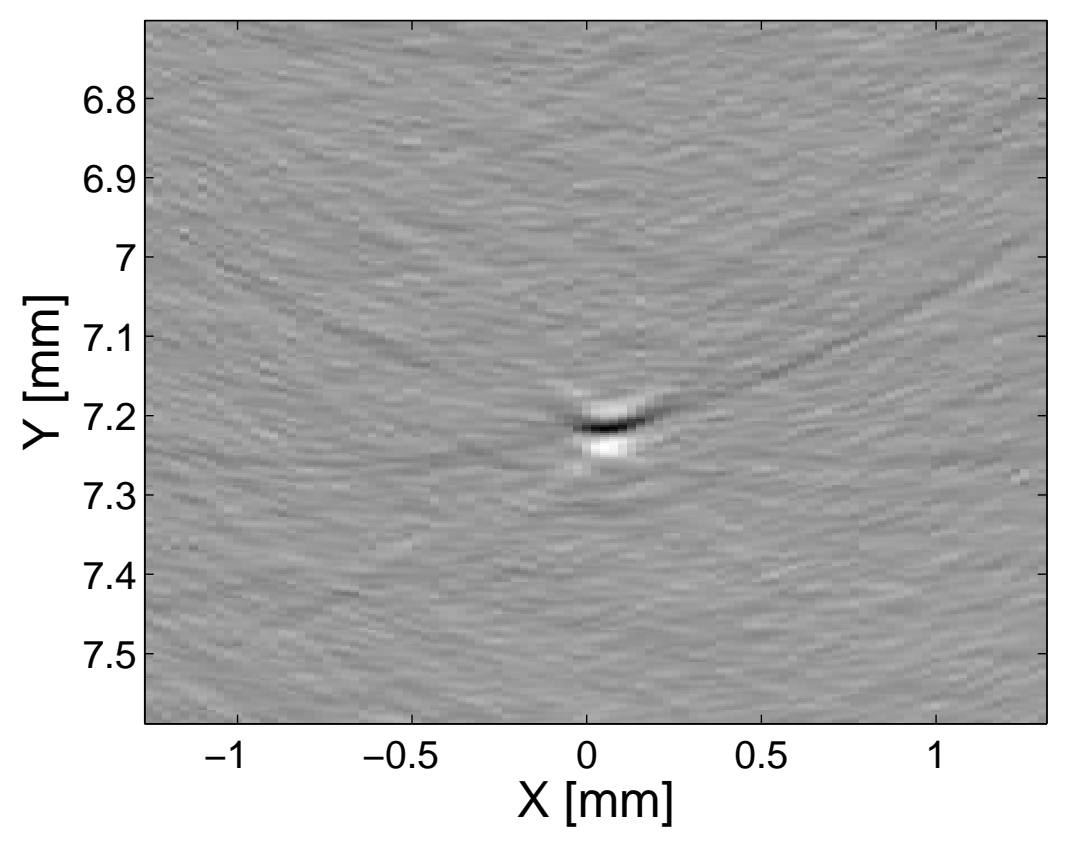

Figure 3. Photoacoustic B-scan of a $6 \mu \mathrm{m}$ carbon fiber in a water-intralipid solution with $\mu_{s}^{\prime}=10 \mathrm{~cm}^{-1}$ at a depth of 7.2 $\mathrm{mm}$.

\section{DISCUSSION}

Because the standard deviation of these pulse amplitude fluctuations were on average $11 \%$ of the mean photoacoustic signal (likely due to electronic noise, and laser pulse-energy fluctuations), we were only statistically sensitive to microvessel amplitude changes greater than $22 \%$. Improved system noise or high-frame-rate averaging may help abate this problem in future studies. Future work should focus on a higher channel-count data acquisition system which can handle streaming of data at a high frame-rate. While the present system offers realtime data acquisition, a realtime beamformer and scan converter is also necessary to operate the system with realtime display. With future improvements, it may be possible to use Eq. 8 to estimate microvessel diameter changes for studies of tissue function and cardiovascular pulse-wave dynamics in the microvasculature.

\section{SUMMARY AND CONCLUSIONS}

A first-of-its kind realtime-acquisition photoacoustic microscopy system based on a high pulse-repetition-rate laser and a high-frequency ultrasound array transducer was demonstrated capable of visualizing microvessels as deep as $3 \mathrm{~mm}$ in living subjects. A $6 \mu \mathrm{m}$ carbon fiber was visualized at a depth of $7.2 \mathrm{~mm}$ in an opticallyscattering medium. The system offers $100 \mu \mathrm{m}$ lateral resolution, $25 \mu \mathrm{m}$ axial resolution, and can image as fast as 83 frames per second. The system shows promise for visualizing photoacoustic amplitude changes due to microvessel diameter changes, which will be important for future diagnostic and scientific studies of microvessel function and cardiac pulse-wave dynamics.

This project was sponsored by NIH grants R01 EB000712 and R01 NS46214. L.V. Wang's e-mail address is lhwang@biomed.wustl.edu.

\section{REFERENCES}

1. K. Maslov, G. Stoica, and L. V. Wang, "In vivo dark-field reflection-mode photoacoustic microscopy," Opt. Lett. 30, pp. 625-627, 2005. 
(a)

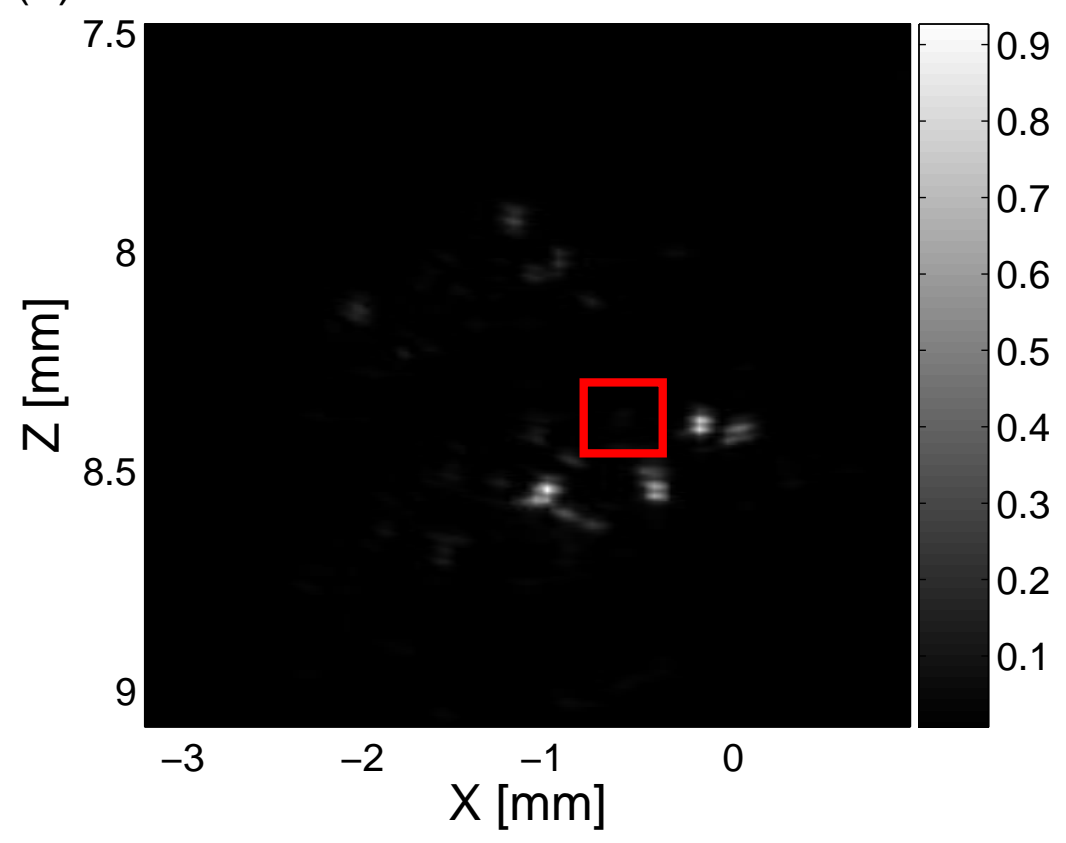

(b)

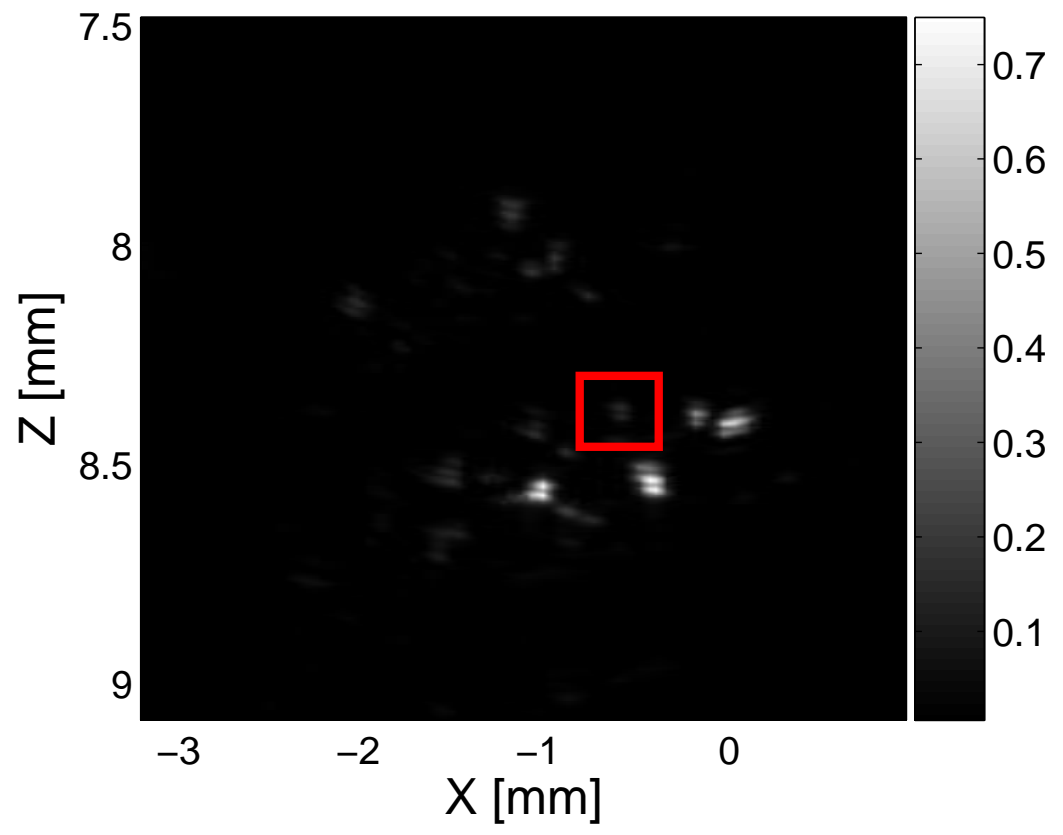

Figure 4. Photoacoustic B-scan of microvessels (a) in diastole and (b) systole as correlated using a pulse-oximeter.

2. H. F. Zhang, K. Maslov, G. Stoica, and L. H. Wang, "Functional photoacoustic microscopy for highresolution and noninvasive in vivo imaging," Nature Biotechnology 24, pp. 848-851, 2006.

3. D. E. Goertz, D. A. Christopher, J. L. Yu, R. S. Kerbel, P. N. Burns, and F. S. Foster, "High frequency color flow imaging of the microcirculation," Ultras. Med. Biol. 26, p. 6371, 2000.

4. D. E. Kruse and K. W. Ferrara, "A new high resolution color flow system using an eigendecomposition-based 
adaptive filter for clutter rejection," IEEE Trans. Ultrason. Ferroelectr. Freq. Contr. 49(12), pp. 1739-1754, 2002.

5. J. J. Niederhauser, M. Jaeger, R. Lemor, P. Weber, and M. Frenz, "Combined ultrasound and optoacoustic system for real-time high-contrast vascular imaging in vivo," IEEE Trans. Med. Imag. 24(4), pp. 436-440, 2005.

6. R. Bitton, R. J. Zemp, M. L. Li, J. Yen, J. Cannata, K. Shung, G. Stoica, and L. V. Wang, "Photoacoustic microscopy with a $30 \mathrm{MHz}$ linear array and receive system," Proc. IEEE Ultrasonics Symposium, 2006.

7. R. J. Zemp, R. Bitton, M. L. Li, K. K. Shung, G. Stoica, and L. V. Wang, "Photoacoustic imaging of the microvasculature with a high-frequency ultrasound array transducer," J. Biomed. Opt., In Press, 2007.

8. P. Carmeliet, "Angiogenesis in life, disease and medicine," Nature 438, pp. 932-936, 2005.

9. M. L. Li, H. F. Zhang, K. Maslov, G. Stoica, and L. H. V. Wang, "Improved in vivo photoacoustic microscopy based on a virtual-detector concept," Optics Lettes 31(4), pp. 474-476, 2006.

10. M. I. Khan, T. Sun, and G. J. Diebold, "Photoacoustic waves generated by absorption of laser radiation in optically thin cylinders," J. Acoust. Soc. Am. 94(2), pp. 931-940, 1993.

11. T. A. Ritter, T. R. Shrout, R. Tutwiler, and K. K. Shung, "A 30-MHz piezo-composite ultrasound array for medical imaging applications," IEEE Trans. Ultrason., Ferroelectr., Freq. Contr. 49(2), pp. 217-230, 2002. 